\title{
Using Mobile Phones to improve Medication Compliance and Awareness for Cardiac Patients
}

\author{
Islam Qudah \\ Faculty of Engineering and IT, \\ University of Technology Sydney \\ ialqudah@it.uts.edu.au
}

\author{
Peter Leijdekkers \\ Faculty of Engineering and IT, \\ University of Technology Sydney \\ Peter.Leijdekkers@uts.edu.au
}

\author{
Valerie Gay \\ Faculty of Engineering and IT, \\ University of Technology Sydney \\ Valerie.Gay@uts.edu.au
}

\begin{abstract}
Improving cardiac patients' medication compliance is a major factor in reducing mortality rate and reducing hospitalization rate. This paper describes a novel medication compliance management system. Its novelty lies in the combination of functionalities that helps the patient to comply with their medication regimen, together with a personal health monitoring system that monitors their health and collects vital signs data using a mobile phone and wireless bio sensors. The system is designed to collect and analyse medication compliance, side effects and symptom responses and transfers the collected data in real time to a web based system for remote monitoring by caregivers and health professionals. Health professionals can use the system to assess the effect of the medication regimen on their patients' health and adapt it to reduce side effects and maximise the patient's wellbeing.
\end{abstract}

\section{Categories and Subject Descriptors}

J.3 [Computer Applications]: Life and Medical sciences - Health

\section{General Terms}

Measurement, Design, Experimentation, Human Factors.

\section{Keywords}

Medication compliance, Cardiac rehabilitation, Ambulatory monitoring, Tele-monitoring,

\section{Introduction}

According to the World health Organization, cardiovascular diseases are the world's number one killer, claiming 17.1 million lives every year [2]. In Australia, cardiovascular diseases kill one person every ten minutes. It is the highest cause of death in Australia with more than $34 \%$ of deaths in 2006 due to cardiovascular disease [14]. Heart Failure and cardiovascular diseases are also the highest cause of mortality in the United States.
Medication compliance is a major factor in reducing the mortality and hospitalization rate [11]. To reduce the cost and burden on public health systems, it is important to bring down unnecessary hospital admissions due to poor medication compliance but also to increase health and medication awareness among people.

Medication compliance rates for patients with a heart failure condition vary widely from $7 \%$ to $90 \%$ for short to midterm treatment [9] [19]. A decline in medication compliance rate is observed for long term heart failure patients which is believed to be the leading cause for subsequent hospital admissions [2] [20] [21]. Some studies suggest that two thirds of rehospitalisation could be prevented by increasing medication compliance rates [22].

A study by Cline [15] found that only $45 \%$ of the patients could name what medication was prescribed to them. Only $50 \%$ of the patients were able to remember the prescribed doses. $36 \%$ were able to remember when the medication was to be taken. Research trials confirmed that noncompliance is common among elderly patients with heart failure condition [10][16].

Common reasons found for patients not complying with their medication regimens are [3][12][17][23]:

- Forgetfulness, the patient does not remember to take their medication.

- Deliberately choosing to disregard the physicians' directives.

- The medication schedule changes.

- Confused by the instructions to take medications.

- Overwhelmed by the number of medications prescribed.

- The fear of side effects or actually experiencing side effects.

- Over/under use of medication or taking the medication at the wrong time.

- Feeling that symptoms disappeared and the medication is no longer necessary.

- Using another person's medication or possibly using old or expired medication.

Age, education and social support are some of the major factors contributing to compliance. A research study in Sweden found that at least $24 \%$ out of 154 elderly cardiac patients needed to be reminded to take their medication. More than $65 \%$ of the patients did not know the benefits of the medication they were taken [13].

Many solutions exist on the market today to tackle medication compliance (see section 2). However, with the wide usage of mobile phones an interesting research question is to investigate whether mobile phones can be used to improve medication compliance for cardiac patients. 
The aim of our research is to design and measure the effectiveness of mobile phones in collecting medication compliance responses from patients and providing health information to patients and health professionals.

This research is part of the Personal Health Monitor research project conducted at the University of Technology, Sydney [PHM]. The Personal Health Monitor is a personalised, intelligent, non-intrusive, real time health monitoring system using wireless sensors and a mobile phone. The wireless sensors can be either attached to the user's body (for example ECG and Accelerometer) or can be external devices, such as a blood pressure monitor or a weight scale, that are used when required. The sensors are Bluetooth enabled or integrated into the mobile phone. On the phone, the Personal Health Monitor software analyses, in real-time, the data received from the sensors. The phone gives immediate feedback and personalised advice to the user based on the analysis of sensor data collected.

In this paper we focus on the integration of a medication compliance system with the use of bio sensors to monitor and provide personalized feedback to patients and health professionals. Section 2 presents an overview of existing medication compliance solutions. Section 3 compares the functionalities offered for each technology and outlines the potential of mobile phones for monitoring medication compliance. Section 4 outlines our novel approach and section 5 discusses the next steps to trial the system in a usability trial at a Sydney hospital.

\section{Existing Medication Compliance Solutions}

Many solutions are available to help improving medication compliance. These solutions can be categorized as pill holders, alarm based pill holders, pill monitoring devices and mobile phone based solutions.

Pill Holders are boxes or containers to carry medications. Usually they are divided into different compartments. The medication is loaded manually in the compartments. The compartments can be labelled for each dosage interval. The differences between pill holders are usually the shape, colour, number of compartments and size. These pill holders are passive i.e. they don't remind patients of their medication, patients need to alert themselves to take their medication.

Alarm Based Aids are active solutions compared to pill holders. Some of these devices consist of compartments to carry medication attached to a timer. When the medication is due an alarm is triggered and the patient needs to open the correct container and take the medication (see Figure 1).

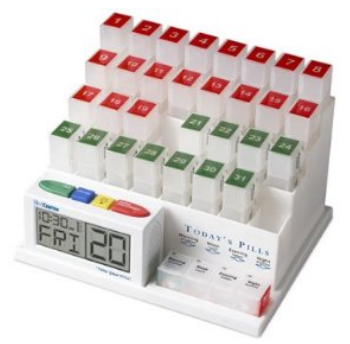

Figure 1: Medcenter ${ }^{\circledR}$ Monthly Medication System with Reminder Alarm
All alarm based medication reminders need to be programmed manually with medication times and names.

Pill Monitoring Devices are home-based medication devices which are sealed (see figure 2). They dispense medication and some of them have voice and text alarms. They can be connected to the internet and alert the patient/caregiver via mobile phone if a dose is missed. They need to be pre-loaded manually with the correct medication.

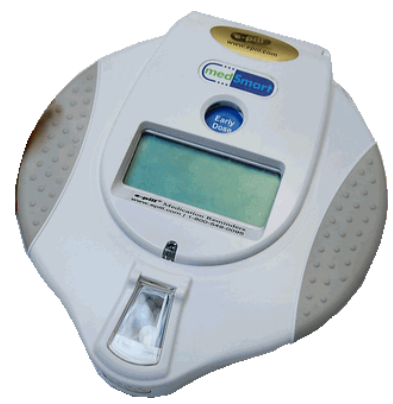

Figure 2: MedSmart ${ }^{\circledR}$ Automatic Medication Pill Dispenser

With the advance of technology and telecommunications, new methods are developed to help collecting medication compliance data. The Med-eMonitor [9] system for example is a portable device with several compartments to store the medication (figure 3 ). It alerts the user when the medication is due and compliance information is transmitted daily via an internet connection to the medical center server for further follow-up by health professionals.

Vitality Inc. [8] is a wireless pill box that will initiate a call and SMS patients reminding them to take their medication. The system does not involve health professionals and no medication compliance information is transmitted to health professionals.

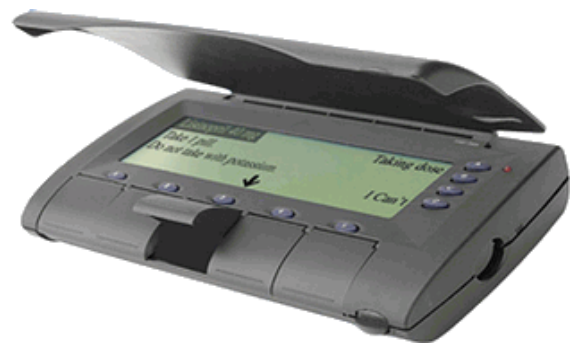

Figure 3: The Med-eMonitor ${ }^{\mathrm{TM}}$ System

Philips [12] offers a medication dispensing service, which is connected to the patient's phone line. When the medication is missed the patient receives a message from the dispensing box.

These are some of the more advanced portable devices that help patients managing their medication regimen. However, these devices are solely designed to measure medication compliance and most of them are home-based devices.

Mobile Phone based solutions are very suitable to manage medication regimens independent of the patient's current location. Two popular commercial solutions are pillPAL [6] and OnTimeRx [4]. These systems require patients to enter manually their medication into the mobile phone or PDA and set the dosage and reminders. These applications are useful for personal use. The 
medication compliance data is not transmitted to health professionals to monitor patient's compliance.

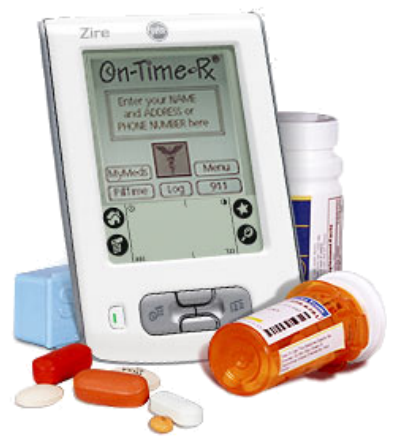

Figure 4: OnTimeRx ${ }^{\circledR}$ medication reminder

Pill Phone and PillBoxer [24] [25] are two solutions to manage medication reminders. The medication regimen is automatically downloaded from a medication pill database into the mobile phone. These solutions allow emailing the medication list and remind patients to take their medication. They are for personal use and there is no interaction with a health professional.

MOBITEL [5] measures vital signs data (blood pressure, pulse and body weight) and transmits it using wireless internet to health professionals on a daily basis. Cardiac patients enter manually the medication dosage on their mobile phone which is then transmitted to the health monitoring centre.

M-PILL [7] reminds patients of their medication and doctor appointments using SMS. The system does not yet support telemonitoring of medication compliance. However, the health professional can send SMS messages to the patient to encourage them to take their medication. Medication compliance data is not transferred to the health professionals to help them in the followup.

eMedonline [25] and MoviTap [26] are two mobile medication management solutions using smart phones, RFID and web services to interact between health professionals and patients. These systems are innovative, but do not analyse the effect of medication on the patient's health.

\section{Technologies versus Functionalities}

Table 1 summarises the various functionalities offered by the different solutions presented in the previous section. Using mobile phones for medication compliance seem to be a logical choice. However, mobile phone solutions do not support the pill holder functionality and it is impossible to actually check whether the medication has been taken on time. An ideal solution would be to combine a pill monitoring device with a mobile phone. For example, a Bluetooth enabled pill monitoring device communicates with a mobile phone application signalling whether the medication has been taken on time. The software on the phone checks the medication regimen and collects feedback from the patient regarding side effects and transmits the collected data to a web based system where health professionals can monitor the medication compliance of their patients. To enhance the system with more accurate feedback, bio sensors can be used to collect physiological data such as blood pressure, weight, blood glucose, oxygen level or even record an ECG to complement the more subjective feedback given by a patient on the use of their medication. For example, Warfarin (to prevent blood clots) can have side effects such as fatigue, joint or muscle aches and many patients would like to reduce/change the dose. If physiological data is collected while altering the dose, better insight is obtained about the effects of dosage change on the patient's wellbeing.

Table 1. Technologies vs. Functionalities

\begin{tabular}{|c|c|c|c|c|}
\hline \multirow[b]{2}{*}{ Functionalities } & \multicolumn{4}{|c|}{ Technology } \\
\hline & Pill Holders & Alarm based Aids & $\begin{array}{l}\text { Pill Monitoring } \\
\text { Devices }\end{array}$ & $\begin{array}{c}\text { Mobile Phone based } \\
\text { Solutions } \\
\end{array}$ \\
\hline Pill holder & $\checkmark$ & $\checkmark$ & $\checkmark$ & $x$ \\
\hline Manual refill & $\sqrt{ }$ & $\sqrt{ }$ & $\sqrt{ }$ & $x$ \\
\hline Portable & $\checkmark$ & $\checkmark$ & $x$ & $\checkmark$ \\
\hline Pill reminder & $x$ & $\sqrt{ }$ & $\checkmark$ & $\checkmark$ \\
\hline Medication schedule modification & Manual & Manual & Manual & $\sqrt{ }$ Remotely \\
\hline Medication and dosages guidance & Manual & Manual & $\sqrt{ }$ & $\sqrt{ }$ \\
\hline Data collection (E.g. Side effects response) & $x$ & $x$ & $\checkmark$ Limited & $\checkmark$ \\
\hline Data Transfer to health centre & $x$ & $x$ & $\checkmark$ Limited & $\checkmark$ \\
\hline Remote monitoring from health centre & $x$ & $x$ & $x$ & $\checkmark$ \\
\hline Record date and time of Pill Bottle opening & $x$ & $\checkmark$ & $\checkmark$ & Manual \\
\hline Medical appointment management & $x$ & $x$ & $x$ & $\sqrt{ }$ \\
\hline $\begin{array}{l}\text { Education and awareness of patients about } \\
\text { medication }\end{array}$ & $x$ & $x$ & $x$ & $\sqrt{ }$ \\
\hline Personalisation & $x$ & $x$ & $\checkmark$ & $\checkmark$ \\
\hline Monitoring of vital health signs & $x$ & $x$ & $x$ & $\sqrt{ }$ \\
\hline
\end{tabular}




\section{A Novel Medication Compliance system}

This section discusses a novel medication system to help patients, caregivers, family, health professionals, pharmacies and health insurance organisations dealing with medication compliance.

The medication system is integrated in the existing Personal Health Monitor (PHM) system which offers a patient a personalised vital signs monitoring solution using a standard mobile phone (Figure 5).

The patient is monitored using a mobile phone and one or more wireless sensors. The phone reminds the patient when measurements or medication is due. Patients can keep track of their blood pressure, weight history and can monitor the progress of their rehabilitation program both on the mobile phone and web based interface on their home computer.

The health professional can remotely manage and check the information about their patients using the web based system. They can remotely personalise and adapt patient reminders and thresholds (e.g. max heart rate set to $120 \mathrm{bpm}$ ) and use the collected information to generate reports.

Changes made by the health professional and data collected on the mobile phone are synchronised periodically, giving both the patient and the health professional access to the latest and most accurate data.

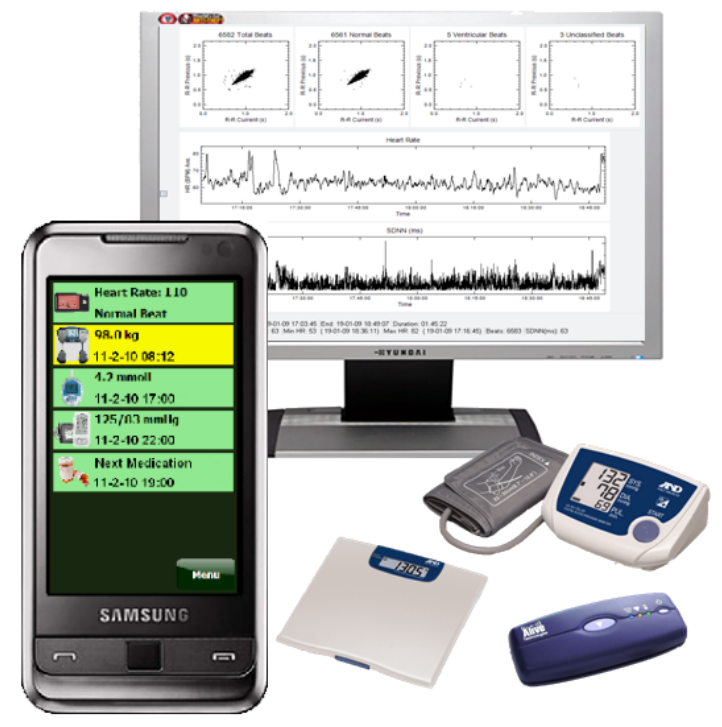

Figure 5: Personal Health Monitor system

The integrated approach enables the collection of complementary information. That is, the vital sign measurements (e.g. blood pressure, heart rate, blood glucose) can complement the selfreported side effects. It also enables complex data mining on medication side effects and compliance.

The PHM system is a web-based system accessible by the different stakeholders. Figure 6 shows a high level view of the PHM medication compliance system showing several stakeholders that interact with the system.

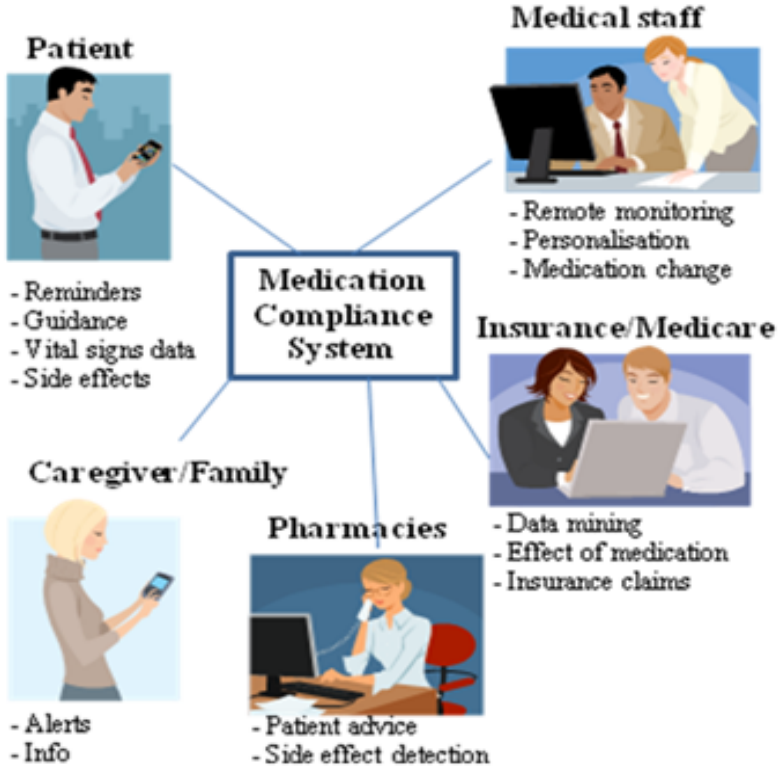

Figure 6: PHM Medication Compliance System Stakeholders

Health professionals can monitor their patients' compliance with their medication intake. Higher compliance rate means that health professionals can make better informed decisions on the effects of the prescribed medication [10]. They can monitor the effect of the medication on their patients and can remotely adapt the medication regimen accordingly. Additionally, health professionals receive indications through the patient's feedback about potential medication interactions that might occur when new medicines are prescribed to the patient.

Family members and caregivers may find it difficult to manage the patient's medication regimen, especially when several medications are prescribed to be taken at different times and dosage [3][12][17]. Family members of an elderly patient may want to know that the medication is taken correctly and be able to personally remind the elderly person of their medication and medical appointments. With the patient's permission, they can subscribe to a service to receive alerts on their mobile phone when medication is due, or have access to view the medication compliance history. If the patient is not compliant for a period of time, an email or SMS can notify caregivers of the problem.

Pharmacies can increase their sales and inform patients better if patients comply with their medication [18]. They receive a warning about potential interactions between different medications used when the patient or health professional updates the medication regimen.

Public and private health insurance companies can connect to the system and request anonymous medication compliance information. This information can help them in developing data mining techniques to assess the effect of increasing/decreasing medication compliance statistics on the number of claims made. Special programs can be introduced to target certain age groups in order to reduce hospitalisation or identify best practice treatments.

Patients are the main beneficiary of the system since it helps them overcome the reasons why they do not comply with their medications. In the introduction, we identified the main reasons 
why patients do not comply with their medication. They can be classified into 4 categories that reflect the need for common functionalities. We integrated these functionalities in the PHM application to help the user complying with their medication.

Issue 1: 'forgetfulness and complex medication regimen, wrong time usage of the medication'. These issues can be solved using better planning aids and reminders. In the PHM application, audio and visual reminders are used to alert patients when it is time to take their medication to overcome forgetfulness (Figure 7, left). The system takes into account the changes made by the health professional and ensures that the medication schedule is always up to date. The application also reminds the patient about their next appointment with their health specialist or to collect new medication at the pharmacy when running low on medication (Figure 7, right).
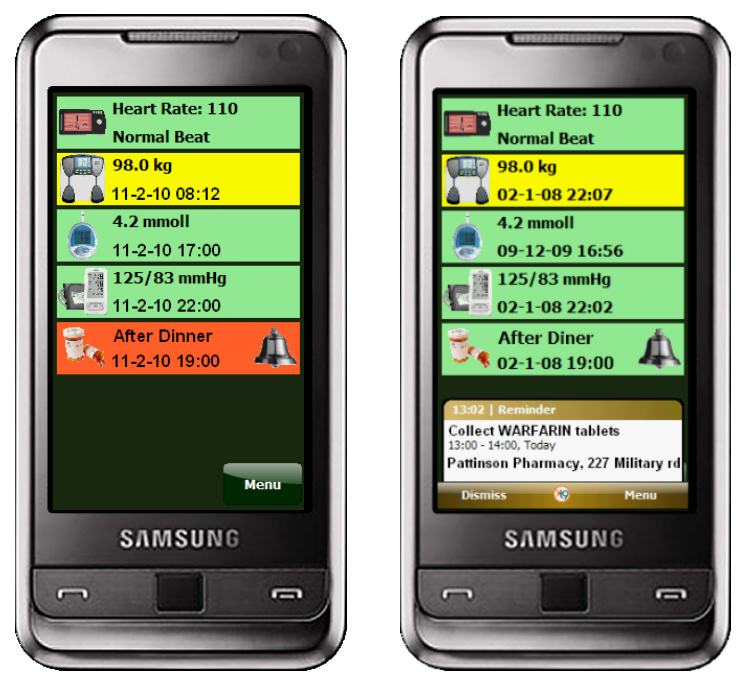

Figure 7: Medication reminders

Issue 2: complex instructions, overwhelming number of medications, over/under use of medication or the use of another person's medication'. These issues can be addressed by offering better guidance to the patient. When the patient gets a reminder, he gets a clear and simple description of the (list of) medication to take at that particular time. For each drug, it includes a photo, the dosage and simple instructions (i.e. take with food) (Figure 8). The patient takes the medication and taps next on the phone to receive the subsequent instructions for the next medication to be taken.

Ideally, the PHM mobile application interacts wirelessly with a pill dispenser device to confirm that the patient has taken the right medicine. Unfortunately no such device exists yet on the market.

Issue 3: 'experiencing side effects'. This issue suggests the need for ways to report, $\log$ and cross check (using sensors) the side effects. Knowing the side effects and medication compliance responses allows health professionals to alter medications accordingly to try decreasing side effects. The patient can provide feedback he experiences at any time or when he is taking the drug (Figure 9). The patient can report different side effects or pains he experiences by answering the simple questions asked by the PHM application. Examples of side effects caused by medication include fatigue, dizziness, rash, mouth swelling, chest pain, fever and shortness of breath.
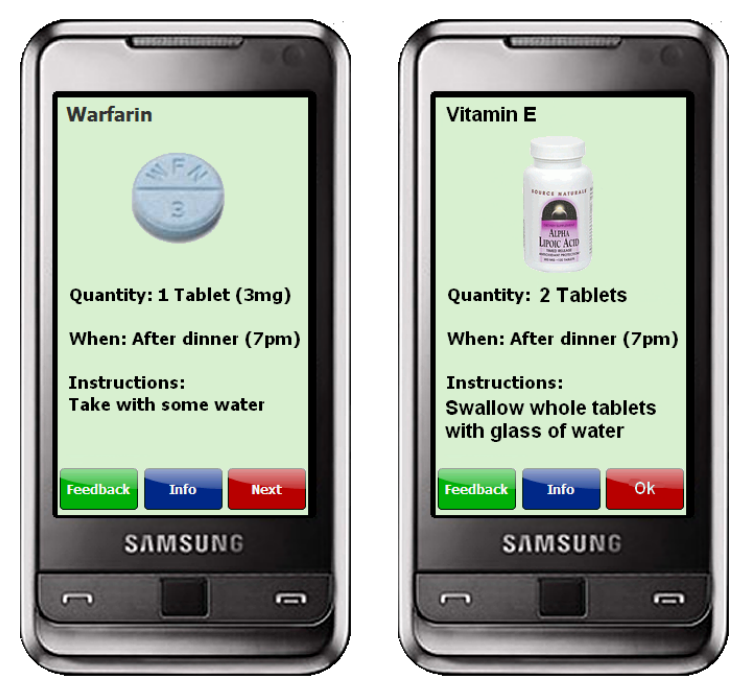

Figure 8: Simple instructions

Issue 4: 'fear of side effects, disregarding the physician's directives or feeling that it is no longer necessary'. These issues suggest a need for more education and information about the medication and health condition. The PHM application has an Info button (Figure 8 Info) directing the patient to an educative link or a common drug database specifying side effects and other information about the medication. It informs patients and caregivers and allows them to post questions to health professionals and receive feedback on their emails about different aspects of their medication.

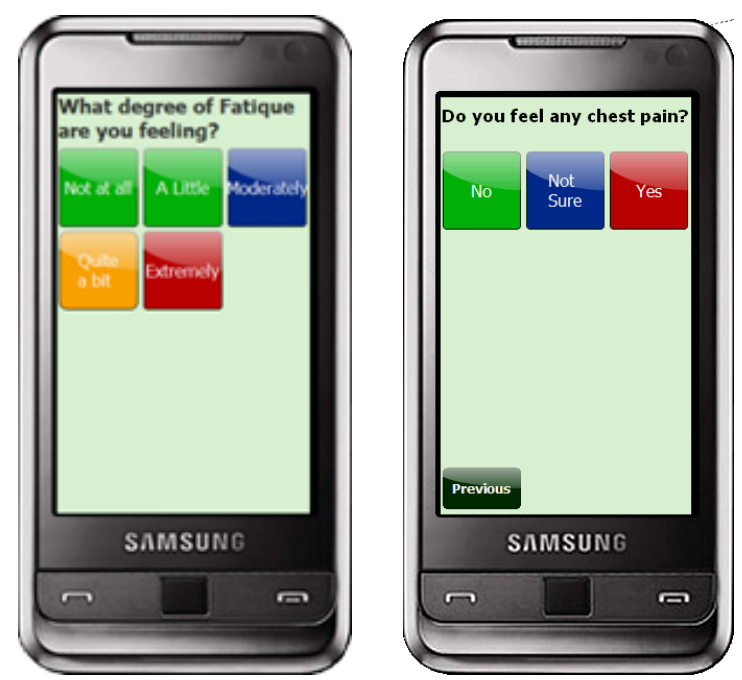

Figure 9: Feedback can be reported to health professionals

We have developed a first version of the medication compliance system for the mobile phone addressing the aforementioned issues. The patient has the PHM application installed on their mobile phone and has also an account with the web based PHM system. The mobile phone connects to the PHM backend wirelessly using $3 \mathrm{G}$ or WiFi and the patient's medication regimen is automatically synchronised with the PHM back end. The feedback of the patient (e.g. side effects, pill taken or forgotten) is 
collected and if necessary, sensors collect vital signs data such as blood pressure, weight and ECG recording.

The PHM web based system is extended with a medication compliance module. Additional information collected for each patient are:

- A list of medication and prescriptions (dose, frequency and history).

- A log of medication compliance data consisting of medications taken/ forgotten and side effect reported by the patient.

- A $\log$ of the interactions between the patient and health professional or Medical web sites.

The patient has also access to general information on medication and possible interactions and side effects.

\section{CONCLUSION}

We are in the process of setting up a clinical trial at the Sydney Royal North Shore hospital to test the potential of the Personal Health Monitor medication compliance system for both patients and health professionals. Other stakeholders such as pharmacies and health insurance companies or not involved in the first phase.

On the patient's side, we intend to incrementally adapt the system to take into account the patient feedback to the medication compliance system. Based on feedback of a previously conducted trial with over 90 patients with the Personal Health Monitor system, we are confident that the user interface is useable by patients of any age. In this trial, however, we would like to understand the impact of reminders on taking medications, as well as, obtaining insight into medication compliance and adherence. We will have a control group not using the Personal Health Monitor medication compliance system to compare the results.

We also want to identify what is missing, or could be improved to motivate patients to comply with their medication. We hope to see health improvement and better patient awareness with respect to their medication.

From the health professional perspective, we would like to get feedback on the different methods used to motivate patients. Many methods have already been tried in measuring medication compliance and assisting both patients and health professionals. However, these methods prove to be expensive in the long term or not effective. The use of mobile phones to help improving medication compliance and monitoring side effects is still a research question and we want to investigate if mobile phones might achieve a better result than conventional methods.

Another goal is to find out what business model works best with our medication compliance system and obtain insight in the costs/benefits of using our system. For example do patients contact health professionals more often or less? If the workload increases is it manageable and cost effective? For example if interactions with health professionals increase but hospitalization rate is reduced, is that economically cost effective?

The main objective of improving medication compliance rate is to reduce mortality and morbidity rates and reduce the cost and burden on the health system, due to preventable hospitalizations. We hope that the integration of the medication compliance module into the Personal Health Monitor suite of health services allows for collecting valuable medication compliance data and side effects responses. We believe that our Personal Health Monitor medication compliance software is of particular interest to patients and health professionals in remote rural areas since it allows them to save travel time to do trivial medical checks like checking ECG rate and to manage complex medication prescriptions.

\section{REFERENCES}

[1] Personal Health Monitor website, viewed 01 December 2009, URL <http://PersonalHealthMonitor.net/>

[2] World Health Organization website, viewed 01 December 2009,URL < http://www.who.int/cardiovascular diseases/en/i ndex.html $>$

[3] Ameliatek website, viewed 20 September, viewed 21 September 2009, $\mathrm{URL}<\mathrm{http}: / /$ www.ameliatek.com/30/medication-errors.html $>$

[4] Ontimerx website, viewed 26 December 2009, $\mathrm{URL}<$ http://www.ontimerx.com/PDA/tourBB.php $>$

[5] Scherr, D. et al., 2009, 'Effect of home-based telemonitoring using mobile phone technology on the outcome of heart failure patients after an episode of acute decompensation: randomized controlled trial', Journal of medical Internet research, vol. 11 , no. 3 , e34.

[6] E-pill website(pillPAL system), viewed 20 December 2009, $\mathrm{URL}<\mathrm{http}: / /$ www.epill.com/pillpal.html>

[7] M-PILL website, viewed 20 December 2009, URL $<$ http://www.m-pill.com/ >

[8] Mobile Tech Today Magazine, 'Next: The Pill Bottle Cap with a Cell Phone', viewed 20 December 2009, URL < http://www.mobile-tech-today.com >

[9] Artinian, N.T., Harden, J.K., Kronenberg, M.W., Vander Wal, J.S., Daher, E., Stephens, Q. \& Bazzi, R.I., 2003, 'Pilot study of a Web-based compliance monitoring device for patients with congestive heart failure', Heart \& Lung: The Journal of Acute and Critical Care, vol. 32, no. 4, pp. 22633.

[10] Claxton, A., Cramer, J., Pierce, C., 2001, ‘A systematic review of the associations between dose regimens and medication compliance' Clinical Therapeutics, vol.23, no.8, pp.1296-1310.

[11] Rasmussen, J., Chong, A., Alter, D., 2007, 'Relationship between adherence to evidence-based pharmacotherapy and long-term mortality after acute myocardial infarction', JAMA, vol.297, no.2, pp.177-186.

[12] Philips Website - Medication mistakes , viewed 24 December2009,URL $<$ http://www.managemypills.com/conte $\underline{\text { nt/Medication errors }>}$ 
[13] Ekman, I., Andersson, G., Boman, K., Charlesworth, A., Cleland, J.G.F., Poole-Wilson, P. \& Swedberg, K. 2006, 'Adherence and perception of medication in patients with chronic heart failure during a five-year randomised trial', Patient education and counselling, vol. 61, no. 3, pp. 348353.

[14] Australia Bureau of Statistics - cardiovascular diseases, viewed 30 December 2009, URL<http://www.abs.gov.au $>$

[15] Cline, C., BYA, I. \& Willenheimer R., 1998, 'Cost effective management programme for heart failure reduces hospitalisation', Heart, vol.80, pp.442-446.

[16] Monane, M., Bohn, R.\& Gurwitz, J., 1994, 'Noncompliance with congestive heart failure therapy in the elderly', Arch Intern Med, vol.154, no.4, pp.433-437.

[17] Caring today website, viewed 28 December 2009 , URL $<$ http://www.caringtoday.com/manage-medications/theimportance-of-medication-compliance $>$

[18] FreshPatents website, 2009, 'Interface system for displaying comprehensive patient medication record', viewed 25 December 2009, URL < http://www.freshpatents.com>

[19] Rich, M.W., Gray, D.B, Beckham, V., Wittenberg, C.\& Luther, P., 1996, 'Effect of a multidisciplinary intervention on medication compliance in elderly patients with congestive heart failure', Am J Med, vol. 101, no. 3, , pp. 270-276.

[20] Miura T., Kojima R., Mizutani M., Shiga Y., Takatsu F. and Suzuki Y., 2001, 'Effect of digoxin noncompliance on hospitalization and mortality in patients with heart failure in long-term therapy: a prospective cohort study',European journal of clinical pharmacology, vol. 57 , no. 1, pp. 77-83
[21] Hope, CJ., Wu, J., Tu, W., Young, J. \& Murray, MD., 2004, 'Association of medication adherence, knowledge, and skills with emergency department visits by adults 50 years or older with congestive heart failure', Am J Health Syst Pharm, vol. 61, no.19, pp. 2043-2049.

[22] Rich, M,., Beckham, V., Wittenberg, C., Leven, CL., Freedland, KE. \& Carney, RM., 1995, 'A multidisciplinary intervention to prevent the readmission of elderly patients with congestive heart failure', $N$ Engl J Med, vol. 333, no. 18 , pp. $1190-1195$

[23] Kidder, D. P., Park, D. C., Hertzog, C., \& Morrell, R. W. 1997, 'Prospective memory and aging: The effects of working memory and prospective memory task load', Aging Neuropsychology and Cognition, vol.4, no. 2, pp. 93-112.

[24] Mobile health news website, viewed 02 January 2010, URL $<$ http://mobihealthnews.com/628/pillbox-vs-pillboxervs-the-pill-phone/>

[25] eMedonline website, viewed 02 January 2010, URL $<$ http://www.emedonline.com/>

[26] MyTap website, viewed 02 January 2010, URL < http://www.mytap.net/> 